\title{
A 0.24pJ/bit, 16Gbps OOK Transmitter Circuit in 45-nm CMOS for Inter and Intra-Chip Wireless Interconnects
}

\author{
Tanmay Shinde \\ Rochester Institute of Technology \\ NY - 14623 USA \\ tvs6972@rit.edu \\ M Meraj Ahmed \\ Rochester Institute of Technology \\ NY - 14623 USA \\ ma9205@rit.edu
}

\author{
Suryanarayanan Subramaniam \\ Rochester Institute of Technology \\ NY - 14623 USA \\ ss4050@rit.edu
}

\author{
Mark Indovina \\ Rochester Institute of Technology \\ NY - 14623 USA \\ maieee@rit.edu
}

\author{
Padmanabh Deshmukh \\ Rochester Institute of Technology \\ NY - 14623 USA \\ psd9321@rit.edu \\ Amlan Ganguly \\ Rochester Institute of Technology \\ NY - 14623 USA \\ axgeec@rit.edu
}

\begin{abstract}
Research in recent years has demonstrated that intra and inter-chip wireless interconnects are capable of establishing energy-efficient data communications within as well as between multiple chips. This paper presents a circuit level design of an energy-efficient millimeter wave (mm-wave) on-off keying (OOK) transmitter suitable for such wireless interconnects in 45-nm CMOS process. The transmitter consists of an NMOS cross-coupled VCO, an OOK modulator and a power amplifier. The transmitter is able to achieve maximum modulation data rate of $16 \mathrm{~Gb} / \mathrm{s}$ at $60 \mathrm{GHz}$ with the output power of $3 \mathrm{dBm}$ consuming a total power of $3.9 \mathrm{~mW}$, which translates to a bitenergy efficiency of $0.24 \mathrm{pJ} / \mathrm{bit}$.
\end{abstract}

\section{KEYWORDS}

Wireless interconnect, mm-wave, transmitter, VCO, OOK modulator, power amplifier, transformer neutralization

\section{ACM Reference Format:}

T. Shinde, S. Subramaniam, P. Deshmukh, M. M. Ahmed, M. Indovina and A. Ganguly. 2018. A 0.24pJ/bit, 16Gbps OOK Transmitter Circuit in 45-nm CMOS for Inter and Intra-Chip Wireless Interconnects. In GLSVLSI '18: 2018 Great Lakes Symposium on VLSI, May 23-25, 2018, Chicago, IL, USA. ACM, NY, NY, USA, 6 pages. https://doi.org/10.1145/3194554.3194575

\section{INTRODUCTION}

To keep up with the demands in computation and communication of platform based computing devices like microservers or embedded systems with multiple multicore chips both on-chip and chip-to-chip communication infrastructures have been evolving at a rapid pace. However, recent trends according to the International Technology Roadmap for Semiconductors (ITRS) predict that the pitch of the I/O interconnects in ICs is not scaling as fast as the gate lengths or pitch of on-chip interconnects. This implies a gap in density and performance of

Permission to make digital or hard copies of all or part of this work for personal or classroom use is granted without fee provided that copies are not made or distributed for profit or commercial advantage and that copies bear this notice and the full citation on the first page. Copyrights for components of this work owned by others than ACM must be honored. Abstracting with credit is permitted. To copy otherwise, or republish, to post on servers or to redistribute to lists, requires prior specific permission and/or a fee. Request permissions from Permissions@acm.org.

GLSVLSI '18, May 16-18, 2018, Chicago, IL, USA

(C) 2018 Association for Computing Machinery.

ACM ISBN 978-1-4503-5724-1/18/05 ..\$15.00

https://doi.org/10.1145/3194554.3194575 traditional I/O systems relative to on-chip interconnections [1]. The wiring complexity of both intra and inter-chip interconnects poses design challenges, crosstalk and signal integrity issues. This has led to the exploration of novel interconnect technologies like photonic interconnect [2] and wireless interconnects [3] as an energy efficient solution for inter and intra-chip data communication. Long-range wireless transmitters and receivers (transceivers) operating in the millimeter-wave (mm-wave) frequencies [4] do not require laying out of physical interconnects making them suitable for a variety of application domains such as embedded system platforms, servers, wearable or flexible electronic devices as well as IoT devices. In addition, such mm-wave transceivers and antennas are designed in CMOS-compatible processes, solutions to the wired interconnect problem. Research in recent years has demonstrated that on-chip and off-chip wireless interconnects are capable of establishing radio communications within a chip as well as between multiple chips and are shown to improve system-level energy efficiency and bandwidth of inter and intra-chip data communications by avoiding or eliminating data communication over long multihop wired paths [3].

For computing platforms with multiple chips, an energy efficient, high bandwidth interconnect fabric is desirable. A multi-gigabit wireless channel has the potential to provide such high performance with high energy efficiency [5]. However, the constant need for lower energy expenditure in data transfer between chips motivates newer designs. Moreover, to be compatible for integration with current and future technology nodes the transceivers need to be designed in advanced technology nodes. This poses a problem to the design of powerefficient circuits as advanced technologies operate in low rail

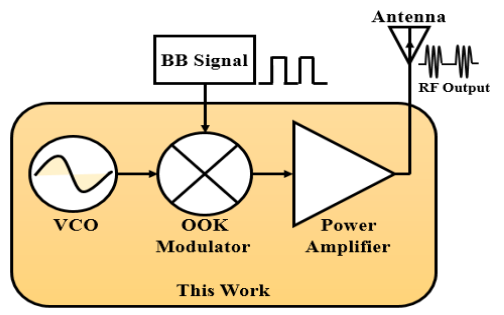

Figure 1: Proposed block diagram of the OOK 


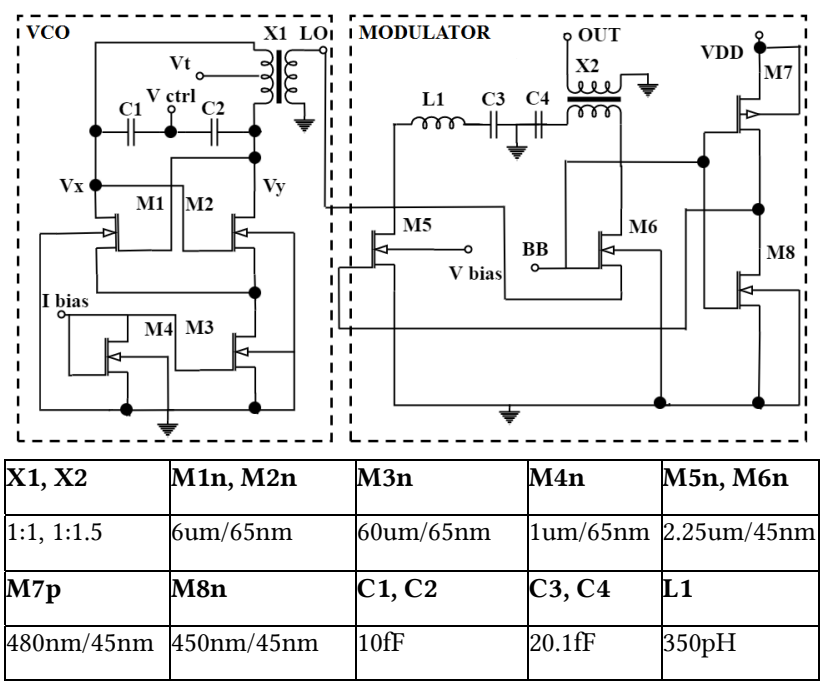

Figure 2: Schematic and component values of the proposed OOK modulator with NMOS cross-coupled

voltages which makes achieving high voltage swings at the output of the transmitter challenging. Therefore in our work we present the circuit design of an ultra-low power CMOS compatible $16 \mathrm{Gbps} \mathrm{mm}$-wave transmitter at $60 \mathrm{GHz}$ in $45 \mathrm{~nm}$ technology node for inter and intra-chip wireless communication. We adopt On-Off Keying (OOK) as the modulation mechanism for its simplicity and low power consumption although it has the lowest bandwidth efficiency. The transmitter proposed in this work consists of $60 \mathrm{GHz}$ Voltage Controlled Oscillator (VCO), an OOK modulator and a power amplifier (PA) as shown in Fig. 1.

\section{RELATED WORK}

In [5], a $60 \mathrm{GHz}$ OOK transmitter that employed a five stage CS power amplifier have been proposed. Though the transmitter provided high amplification gain, it came with a cost of increased power dissipation and overall noise due to higher number of components. An energy dissipation of $114 \mathrm{pJ} / \mathrm{bit}$ along with low data rate of $2.5 \mathrm{Gbps}$ over a $40 \mathrm{~mm}$ communication range made the transmitter inefficient for future low power high performance intra and inter-chip communication. A $9.5 \mathrm{pJ} / \mathrm{bit}$, 6Gbps ASK transmitter operating at $43 \mathrm{GHz}$ was proposed in [6]. However, the use of Si-Ge process makes the integration of this transceiver circuit with a standard CMOS chip challenging. In [7] a data rate of $11 \mathrm{Gbps}$ with $10^{-11}$ Bit Error Rate (BER) was achieved with a cost of $6.4 \mathrm{pJ} / \mathrm{bit}$ energy efficiency for the transmitter designed in $40 \mathrm{~nm}$ node. However, the transmitter communication was limited to $14 \mathrm{~mm}$ and therefore cannot be used for energy efficient chip-to-chip communication. In [8], author proposed OOK transceiver for on-chip communication as the transmitter provides $60 \mathrm{GHz}$ OOK modulation at $3.5 \mathrm{Gbps}$ maximum data rate. In [9], the author proposed a multi modal transmitter with a highest data rate of $20 \mathrm{Gbps}$ for free space communication. As the highest reliable communication distance was limited to $5 \mathrm{~mm}$, the transmitter can be used only for on-chip communication. The work proposed in [10] demonstrated a transmitter suitable for a maximum communicating distance of $100 \mathrm{~mm}$ at $10.7 \mathrm{Gbps}$ data rate with $3.45 \mathrm{pJ} /$ bit efficiency. However the transmitter design was faced with the challenge of integrating the on-board Yagi-Uda antenna on a non-silicon substrate. An OOK transmitter capable of communicating within a range of $20 \mathrm{~mm}$ with a BER of $10^{-15}$ and having an energy efficiency of $1.2 \mathrm{pj} / \mathrm{bit}$ and $16 \mathrm{Gbps}$ data rate has been proposed in [11] as a solution for on-chip communication. However, its performance for chip-to-chip communication is not evaluated. In this work, we present the design of an OOK transmitter circuit for intra-chip as well as long range inter-chip communication for future high performance multichip computing platforms.

\section{OOK TRANSMITTER}

The scenario of inter-chip and intra-chip wireless mm-wave links requires extremely low energy consumption to be competitive with high-speed serial $\mathrm{I} / \mathrm{O}$ and other emerging technologies like photonic interconnects. Therefore, an OOK transmitter is preferred for its energy-efficiency. Fig. 1 shows the block diagram of proposed OOK transmitter consisting of a VCO, modulator and PA. In this section we discuss the design approaches for these components of the $60 \mathrm{GHz}$ OOK transmitter.

\subsection{Voltage Controlled Oscillator}

The VCO is responsible for generating the $60 \mathrm{GHz}$ RF carrier wave for the OOK modulator. The maximum single-ended voltage swing with minimum phase noise can be achieved by an NMOS cross-coupled VCO as it has comparatively less phase noise compared to the other oscillators such as ring or LC oscillator [12]. Moreover, it takes less active area compared to Colpitts oscillator. In addition to providing a higher output swing, this design also supports wide tuning range. In the cross coupled NMOS oscillator shown in Fig 2, the positive feedback is given via the M1, M2 transistors and the LC tank circuit. Each of the transistors creates a $180^{\circ}$ phase shift at its output. The control voltage i.e. $\mathrm{V}_{\mathrm{ctrl}}$ is set at $100 \mathrm{mV}$ and given to both the capacitors as shown in Fig. 2. Current biasing is used for the VCO and the required current is generated via a simple NMOS current mirror for a reference input of $10 \mathrm{uA}$. The startup time is a critical part in the oscillator design. To avoid the startup time from limiting the data rate we do not use the $\mathrm{VCO}$ as a modulator. Therefore, the output of the VCO, the Local Oscillator (LO) signal, is coupled to a separate OOK modulator input via a transformer, $\mathrm{X} 1$ with a $1: 1$ turns ratio to ensure symmetrical swing at the input of the modulator. We adopt transformer matching technique as it reduces overall power consumption by eliminating buffers or coupling capacitors. The two transistors of the VCO, M1 and M2, form a differential pair where only one of them is on at a given time degrading the trans-conductance of the pair. Therefore, we increase the width of the transistors M1 and M2 sufficiently, to provide high transconductance and drive the OOK modulator. 


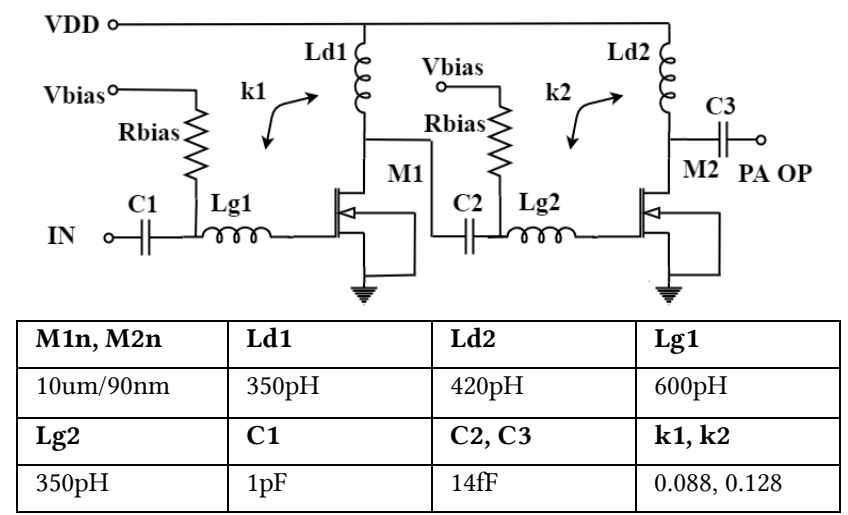

Figure 3: Schematic and component values of the proposed power amplifier.

\subsection{OOK Modulator}

The design of a modulator for high speed inter and intra-chip communication poses a significant challenge on the energy efficiency of the transmitter as power consumption increases with data rate. In the proposed modulator shown in Fig. 2, the modulating M6 NMOS transistor is driven by the Base Band signal (BB). The BB signal turns on and off M6 by charging up the gate terminals above the threshold voltage $V_{\text {th }}$ of the NMOS transistor. For logic HIGH at BB, NMOS M6 gets switched on and it passes the LO signal connected to the source of M6 towards its drain and the primary winding of the transformer X2. Effectively, M6 works a pass transistor, passing the carrier signal from its source to drain when the BB signal is logic $\mathrm{HIGH}$ and blocking it when the BB signal is logic LOW. When BB signal has a logic LOW value, all charge across primary side of $\mathrm{X} 2$ needs to be discharged. This charge gets discharged through NMOS transistor M5. Similar to the transformer X1 coupling the VCO to the modulator, another 1:1.5 transformer, X2 is designed to obtain single ended output from the modulator and for impedance matching of the modulator output with the input of the PA. The primary side of transformer $\mathrm{X} 2$ is connected to ground through capacitor C4. Capacitor C4 and C3 cancels the effect of the imaginary part of the impedance due to the inductor coil of the transformer. The body biasing technique is used with NMOS M5 for adjusting the threshold voltage to get symmetrical signal across $0 \mathrm{~V}$ at the secondary coil of X2.

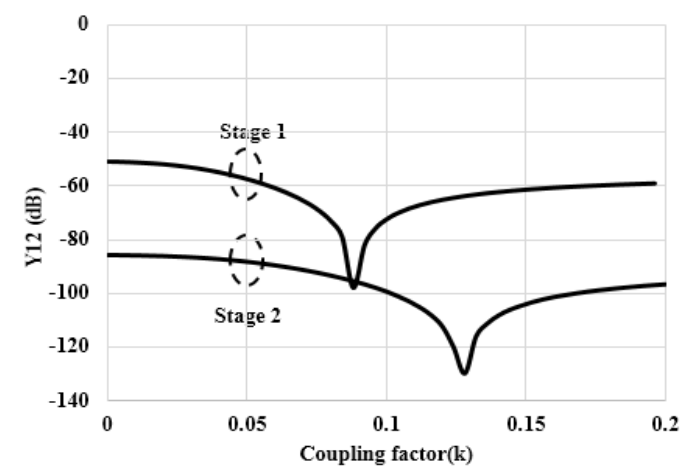

Figure 4: Simulated $\mathrm{Y}_{12}$ for PA versus coupling factor,

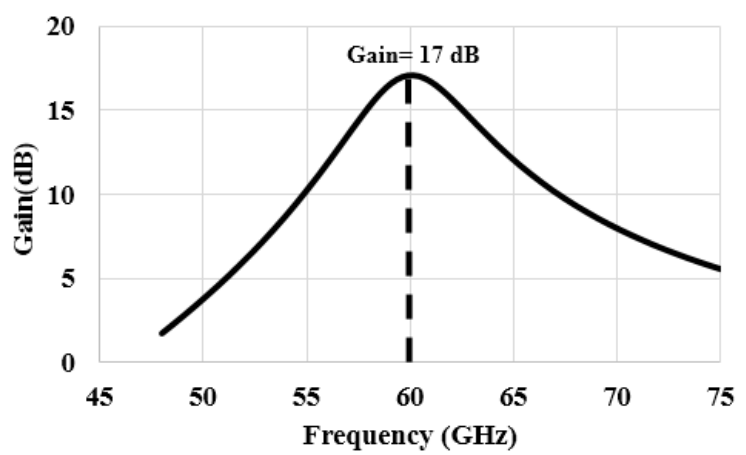

Figure 5: Simulated VCO gain.

\subsection{Two Stage PA}

In the transmitter, power amplifier is one of the power-hungry blocks that affects the overall efficiency of the transmitter. Cascode structures, which are commonly used to obtain high gains at lower frequencies, are not suitable for high frequency applications as the parasitic capacitances become dominant and the stacking of transistors reduces the voltage headroom in advanced technology nodes operating at low rail voltages [13]. Therefore, in our design, a two-stage Common Source (CS) topology is used as shown in Fig. 3 as it provides high frequency response and larger voltage swing compared to cascode designs. At high frequency of operation, degradation in the performance of power amplifier is caused by the drain-to-gate parasitic capacitor $\mathrm{C}_{\mathrm{gd}}$, which introduces a direct signal path between output and input. This drain-to-gate feedback degrades reverse isolation and can directly lead to instability of the amplifier. Moreover, as any change in output impedance affects the input impedance through $\mathrm{C}_{\mathrm{gd}}$, it significantly affects frequency response and output impedance matching of the transmitter. The two-stage PA proposed in this work uses a CS topology with drain-to-gate transformer-feedback neutralization technique which creates an additional signal path that neutralizes the current flow through $C_{g d}[11]$. The transformers are formed using inductors at the gate and drain terminals of M1 ( $\operatorname{Lg} 1$ \& Ld1) and M2 (Lg2 \& Ld2) as shown in Fig. 3 with coupling factors, $\mathrm{k} 1$ and $\mathrm{k} 2$ respectively. The values of the coupling factors are chosen such that they minimize the reverse transformation of the two stages of the amplifier, $\mathrm{Y}_{12}=$ $\frac{I_{g s}}{V_{d s}} \mid V_{i n}=0$. Fig. 4 plots the $Y_{12}$ for the two CS stages of the PA and shows that the optimal values of the coupling factors, $\mathrm{k} 1$ and $\mathrm{k} 2$ are 0.088 and 0.128 respectively. The inductors $\mathrm{Ld} 1$ and $\mathrm{Ld} 2$ at the drain of the transistors M1 and M2 resonate with the drain capacitances. This increases the overall gain and bandwidth at the center frequency. Transistor M1 and M2 are biased using a voltage divider based biasing circuit. To complete the biasing, the DC blocking capacitor $\mathrm{C} 1$ and $\mathrm{C} 2$ are placed to prevent the upsetting of the gate to source bias of M1 and M2. The value of $\mathrm{C} 1$ is chosen to be $1 \mathrm{pF}$. C2 and C3 cancels imaginary impedance effect of drain inductors Ld1 and Ld2 on the output side of both stages. Hence, it has negligible reactance at the center frequency. The output of the power amplifier is matched with $50 \mathrm{Ohms}$ by using $\mathrm{L}$ match impedance matching technique. 


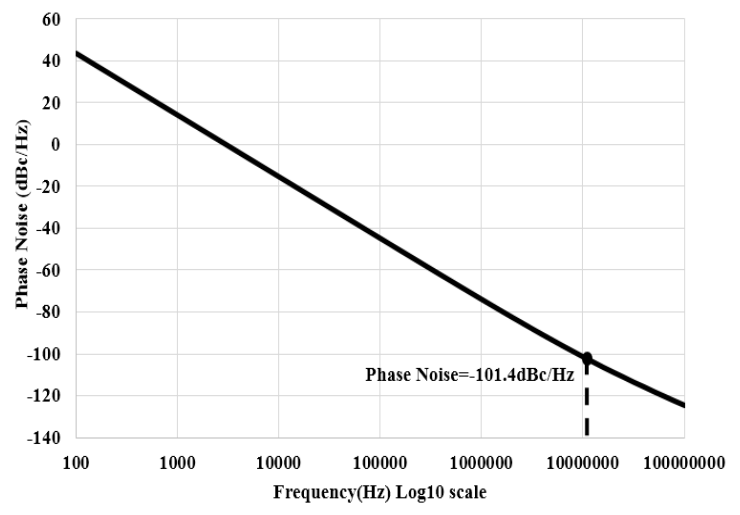

Figure 6: Simulated phase noise of VCO.

\section{RESULTS AND SIMULATION}

The proposed transmitter is designed in 45nm CMOS technology and the simulation is carried out using Cadence Virtuoso Analog Design Environment (ADE). In this section we present the characterization of the $\mathrm{VCO}$, the modulator, the $\mathrm{PA}$ and the overall OOK transmitter.

\subsection{Characterization of the VCO}

In this section, we characterize the VCO for frequency response and phase noise. We consider the frequency range from $45 \mathrm{GHz}$ to $75 \mathrm{GHz}$ for the characterization of the VCO. Fig. 5 shows the frequency response of the VCO. From the figure, it can be observed that a peak-gain of $17 \mathrm{~dB}$ is achieved for the VCO centered at $60 \mathrm{GHz}$ signifying a carrier frequency of $60 \mathrm{GHz}$. Since the oscillator needs to be triggered initially for its proper functionality in the simulation environment, one of the tank capacitors was initialized with $1 \mathrm{nV}$.

The frequency stability is an important concern for the VCO and ideally, it should be immune to the random frequency fluctuations. The measure of these fluctuations is characterized by the phase noise. Phase noise is defined as the ratio of power in one phase modulation sideband per $\mathrm{Hz}$ bandwidth, at an offset of $f \mathrm{~Hz}$ away from the carrier, to the total signal power. The

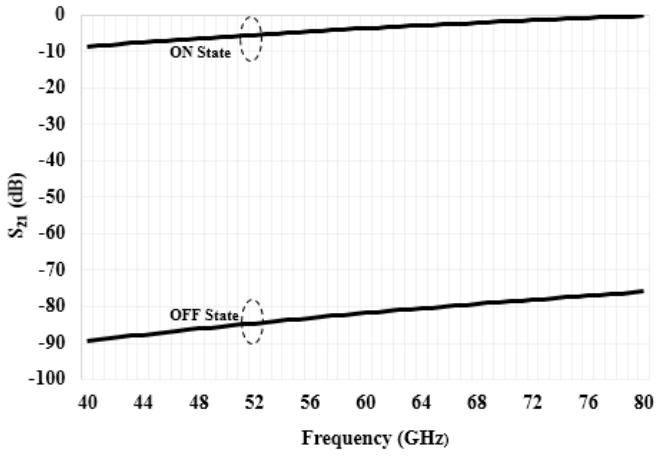

Figure 7: Simulated On-Off gain of the OOK

phase noise evaluated at an offset of $10 \mathrm{MHz}$ is $-101.4 \mathrm{dBc} / \mathrm{Hz}$ as shown in Fig. 6. The VCO consumes a maximum steady-state current of $1.1 \mathrm{~mA}$ from a $1 \mathrm{~V}$ supply.

\subsection{Characterization of the OOK Modulator}

An ideal OOK modulator should not have any output power in the off-state (when the BB input is logic LOW). However, due to non-ideal effects such as leakage, the output power is not completely zero in the off-state. Therefore, a high on-off gain is desirable for reliable detection at an OOK receiver. Here, we characterize our OOK modulator in terms of the on-off steadystate gain. The on-off steady-state gain can be defined as the difference between the steady-state gains of the modulator for the two valid logic levels of the $\mathrm{BB}$ input. At $60 \mathrm{GHz}$ the modulator has an "on" and "off" state gain of $-3.6 \mathrm{~dB}$ and $-81.6 \mathrm{~dB}$ respectively as shown in Fig. 7. Therefore, the on-off steady state gain is above $78 \mathrm{~dB}$ for the frequency ranges from $45 \mathrm{GHz}$ to $75 \mathrm{GHz}$. With this gain the valid logic levels can be detected with a transmitter output power penalty of less than $1 \mathrm{~dB}$ [11].

\subsection{Characterization of the PA}

In this section, we characterize the PA in the OOK transmitter in terms of S-parameters, and Power Added Efficiency (PAE). First, we extract the S-parameter of the PA. At high frequencies i.e.

Table 1: Performance Comparison of mm-wave OOK Transmitter

\begin{tabular}{|c|c|c|c|c|c|c|c|}
\hline References & This Work & {$[11]^{*}$} & {$[7]$ * } & {$[6] *$} & {$[10] *$} & {$[5]$ * } & {$[8] *$} \\
\hline Technology node & 45-nm CMOS & 65-nm CMOS & 40-nm CMOS & 180-nm SiGe & 90-nm CMOS & 90nm CMOS & 90nm CMOS \\
\hline Carrier Frequency & $60 \mathrm{GHz}$ & $60 \mathrm{GHz}$ & $57 \mathrm{GHz}$ & $43 \mathrm{GHz}$ & $60 \mathrm{GHz}$ & $60 \mathrm{GHz}$ & $60 \mathrm{GHz}$ \\
\hline Modulation & OOK & $\mathrm{OOK}$ & ASK & ASK & OOK & $\mathrm{OOK}$ & OOK \\
\hline $\begin{array}{ll}\begin{array}{l}\text { Maximum Data } \\
\text { Rate }\end{array} \\
\end{array}$ & $16 \mathrm{~Gb} / \mathrm{s}$ & $16 \mathrm{~Gb} / \mathrm{s}$ & $11 \mathrm{~Gb} / \mathrm{s}$ & $6 \mathrm{~Gb} / \mathrm{s}$ & $10.7 \mathrm{~Gb} / \mathrm{s}$ & $2.5 \mathrm{~Gb} / \mathrm{s}$ & $3.5 \mathrm{~Gb} / \mathrm{s}$ \\
\hline $\begin{array}{l}\text { Power } \\
\text { Consumption }\end{array}$ & $3.9 \mathrm{~mW}$ & $19 \mathrm{~mW}$ & $29 \mathrm{~mW}$ & $57 \mathrm{~mW}$ & 31.mW & $183 \mathrm{~mW}$ & $156 \mathrm{~mW}$ \\
\hline Supply Voltage & $1 \mathrm{~V}$ & $1 \mathrm{~V}$ & $1.1 \mathrm{~V}$ & $1.6 \mathrm{~V}$ & $0.8 / 1.8 \mathrm{~V}$ & $1.2 \mathrm{~V}$ & N/A \\
\hline RF output P1dB & $-3 \mathrm{dBm}$ & $1.5 \mathrm{dBm}$ & $-2 \mathrm{dBm}$ & $-1.5 \mathrm{dBm}$ & $5.1 \mathrm{dBm}$ & $5 \mathrm{dBm}$ & $2 \mathrm{dBm}$ \\
\hline $\begin{array}{l}\text { Bit-Energy } \\
\text { Efficiency }\end{array}$ & $0.24 \mathrm{pJ} / \mathrm{bit}$ & $1.2 \mathrm{pJ} / \mathrm{bit}$ & $2.6 \mathrm{pJ} / \mathrm{bit}$ & $9.5 \mathrm{pJ} / \mathrm{bit}$ & $2.9 \mathrm{pJ} / \mathrm{bit}$ & $73.2 \mathrm{pJ} / \mathrm{bit}$ & $44.6 \mathrm{pJ} / \mathrm{bit}$ \\
\hline Active-Foot Print & N/A & $0.077 \mathrm{~mm}^{2}$ & $0.06 \mathrm{~mm}^{2}$ & $0.25 \mathrm{~mm}^{2}$ & $0.15 \mathrm{~mm}^{2}$ & $0.43 \mathrm{~mm}^{2}$ & $\mathrm{~N} / \mathrm{A}$ \\
\hline FoM & 2.055 & 1.19 & 0.24 & 0.07 & 1.12 & 0.04 & 0.04 \\
\hline
\end{tabular}




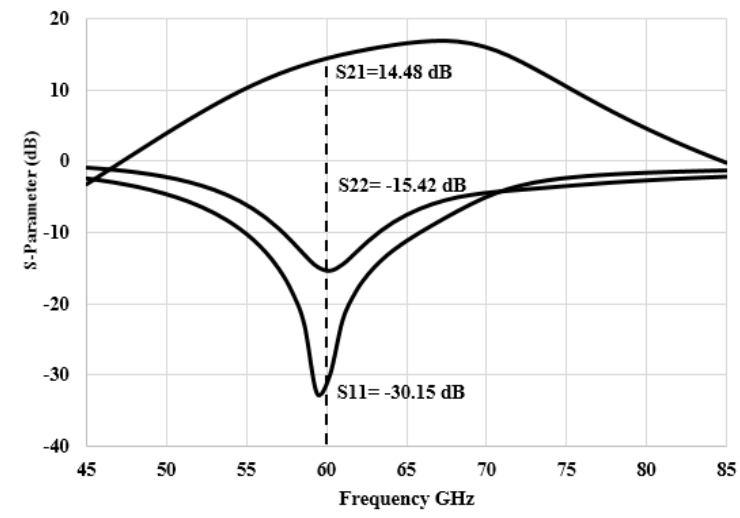

Figure 8: Simulated S-parameters of PA.

$60 \mathrm{GHz}$, it is important to consider the S-parameters of the circuit as it describes the input-output relationship between the terminals of the circuit. A two-port network is setup for the S-parameter extraction. Both the input and output ports are matched to $50 \mathrm{Ohm}$ impedance to extract the S-parameters. Fig. 8 shows the $S_{11}, S_{22}$, and $S_{21}$ for the proposed PA. The $S_{11}$ is the ratio of reflected power to the incident power at the input port and is known as reflection coefficient or return loss. For the proposed PA design the value of reflection coefficient, $S_{11}$ is found to be $-30.15 \mathrm{~dB}$ at $60 \mathrm{GHz}$. Therefore, we conclude that the input is matched well with the $50 \mathrm{Ohm}$ antenna impedance and hence we find negligible reflections at the input at $60 \mathrm{GHz}$. Similarly, $\mathrm{S}_{22}$ is the ratio of reflected power to the incident power on the output port and it is found to be $-15.42 \mathrm{~dB}$ at $60 \mathrm{GHz} . \mathrm{S}_{21}$ is the ratio of output power to the input power and represents the power gain for a well-matched PA. From Fig. $8 S_{21}$ is found to be $14.48 \mathrm{~dB}$.

The power added efficiency (PAE) is defined as the percentage ratio of difference between output and input power over total DC power. Hence it signifies the amplification factor of the PA. Fig. 9 shows the simulated PAE versus input power of the PA at $60 \mathrm{GHz}$. It shows the output power at $1 \mathrm{~dB}$ compression point is $3 \mathrm{dBm}$. Maximum simulated PAE is found to be $17 \%$ at $-3 \mathrm{dBm}$ of output power. Overall, the PA draws $2.2 \mathrm{~mA}$ from a $1 \mathrm{~V}$ supply.

\subsection{Characterization of the Overall Transmitter}

Here, we characterize whole OOK transmitter in terms of data rate, total DC power consumption and transmitted power and bit

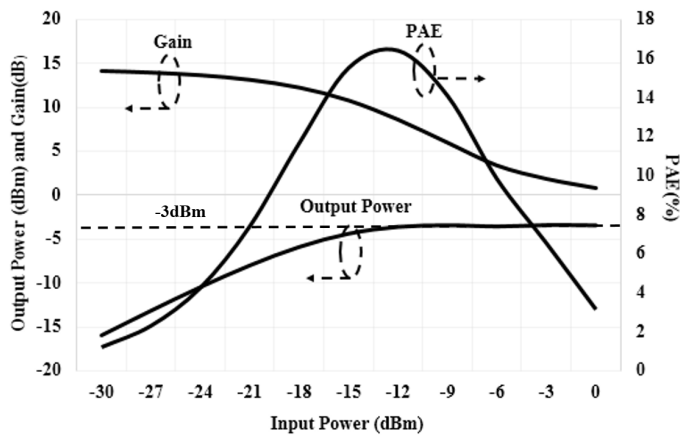

Figure 9: Simulated gain, output power and PAE of the PA versus input power at $60 \mathrm{GHz}$. energy-efficiency. To characterize the OOK transmitter the input and output impedances of PA are matched with the output impedance of OOK modulator and that of a standard antenna impedance of $50 \mathrm{Ohms}$ respectively.

The waveform shown in Fig. 10 (a) shows the amplified OOK modulated signal at the output of PA with a pseudorandom sequence of logic 0 s and $1 \mathrm{~s}$ at baseband signal input. As demonstrated 10 bits (1110101001) are modulated between 1ns and $1.625 \mathrm{~ns}$ indicating a data rate of $16 \mathrm{Gbps}$. From Fig. 10(a) the output waveform indicates a $450 \mathrm{mVpp}$ which translates to $3 \mathrm{dBm}$ output power and is achieved with a total DC power consumption of $3.9 \mathrm{~mW}$. This implies a bit energy efficiency of $0.24 \mathrm{pJ} / \mathrm{bit}$ at $16 \mathrm{Gbps}$. The designed OOK transmitter is compared with other existing designs in comparable technology nodes. For a comprehensive comparison, we use Figure-of-Merit (FoM), which is given by

$$
F O M\left(m W \cdot \frac{\text { bit }}{p J}\right)=\frac{\text { Data Rate }(G b p s) \cdot O P_{1 d B}(m W)}{P_{D C}(m W)} .
$$

Where $P_{D C}$ is the power consumption and $O P_{1 d B}$ is the $1-\mathrm{dB}$ compression point at the output of the transmitter. In Table 1 we compare our OOK transmitter with other transmitters designed for similar applications with respect to important performance metrics. While our design outperforms all the existing works it is to be noted that all the other works are post-layout or postfabrication results while ours is circuit-level characterization.

\subsection{Corner Analysis}

The corner analysis is performed to obtain the performance of the transmitter over different operating voltages and temperatures. The analysis is performed for SS, TT, FF corners over $0.9,1,1.1 \mathrm{~V}$ and 125 , $25,0^{\circ} \mathrm{C}$ operation voltages and temperatures respectively. The
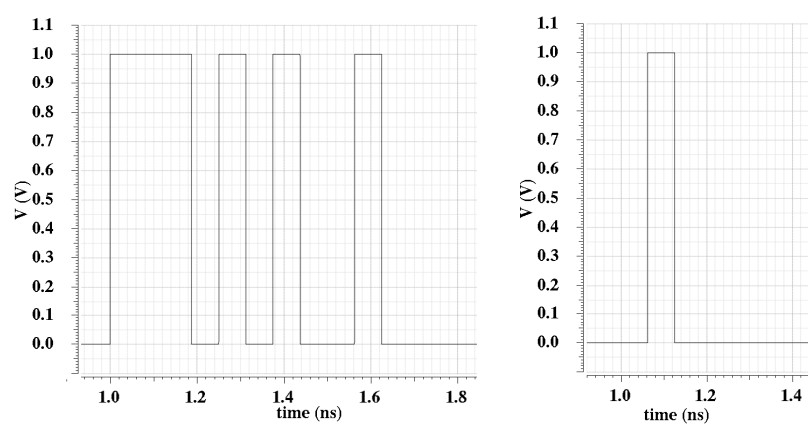

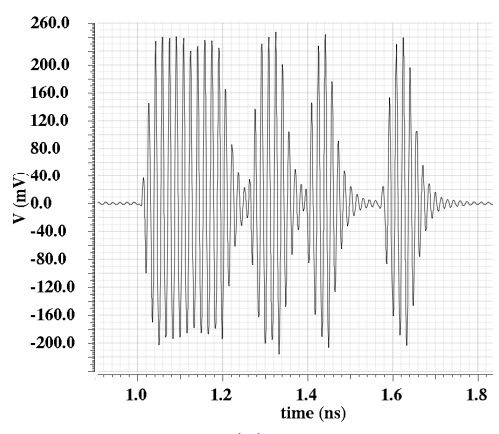

(a)

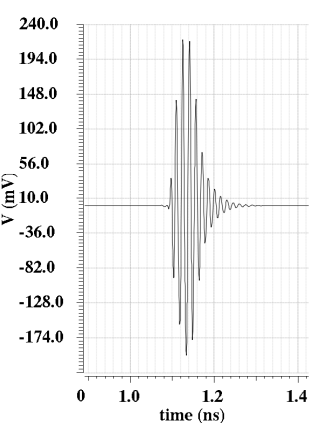

(b)
Figure 10: BB signal and PA output for (a) pseudo-random sequence and (b) lone pulse analysis. 
Table 2: Performance of Transmitter at different corners

\begin{tabular}{|l|l|l|l|l|l|}
\hline Corners & $\begin{array}{l}\text { Voltag } \\
\text { e (V) }\end{array}$ & Temp.(C) & $\begin{array}{l}\text { Speed } \\
\text { (Gbps) }\end{array}$ & $\begin{array}{l}\text { Power } \\
(\mathbf{m W})\end{array}$ & $\begin{array}{l}\text { RooK } \\
(\mathbf{d B})\end{array}$ \\
\hline SS & 0.9 & 125 & 16 & 1.399 & 15.24 \\
\hline TT & 1 & 25 & 16 & 2.064 & 16.54 \\
\hline FF & 1.1 & 0 & 16 & 2.903 & 18.15 \\
\hline
\end{tabular}

characterization of OOK transmitter at the various corner cases is shown in Table 2 with respect to total power consumption and on-off ratio of the overall OOK transmitter, RoOK while operating at a data rate of $16 \mathrm{Gbps}$.

\subsection{Link Budget Analysis}

In [11] the path loss for a $20 \mathrm{~mm}$ intra-chip link is shown to be around $26 \mathrm{~dB}$. In [3] it is shown that the longest distance for an inter-chip link is $59 \mathrm{~mm}$ resulting in a path loss of about $35 \mathrm{~dB}$. Here, we evaluate the BER corresponding to typical intra and inter-chip communication distances. The received signal power is

$$
P_{R}=P_{T}-P L \text {. }
$$

Here, $P_{R}$ and $P_{T}$ are the received and transmitted power respectively and $P L$ is the path loss. We assume a non-coherent OOK receiver as these are most power efficient. LNAs used in typical non-coherent OOK receivers have a noise figure, NF of $10 \mathrm{~dB}$ or less [11]. The Noise Floor, NFloor of such a receiver is,

$$
N_{\text {Floor }}=10 \log (k T)+10 \log (B W)+N F .
$$

Where, $\mathrm{k}$ is the Boltzmann constant, $\mathrm{T}$ is the absolute temperature and BW is the bandwidth. Therefore, from (3) the NFloor of the receiver is $-67.8 \mathrm{dBm}$ at $300 \mathrm{~K}$ for a $B W$ of $16 \mathrm{GHz}$. However, in addition to thermal AWGN, Inter-symbol Interference (ISI) due to bandwidth limitation of the transmitter can add interference noise. As can be seen from the lone-pulse analysis in Fig. 10(b) the transmitter output is $450 \mathrm{mVpp}$ and $67 \mathrm{mVpp}$ when it is transmitting a ' 1 ' and a ' 0 ' respectively when measured at the center of the pulses. Therefore, the on-off ratio of overall OOK transmitter, ROOK is $16.54 \mathrm{~dB}$. The value of ISI noise, NISI is dependent on path loss and is given by,

$$
N_{I S I}=P_{T}-R_{O O K}-P L .
$$

The received Signal to Interference and Noise Ratio (SINR) is,

$$
\text { SINR }=P_{R}-N_{\text {Floor }}-N_{I S I} \text {. }
$$

For non-coherent OOK demodulation, the BER is given by,

$$
B E R_{O O K}=\frac{1}{2} \exp \left(-\frac{1}{2} \operatorname{SINR}\right)+\frac{1}{4} \operatorname{erfc}\left(\sqrt{\frac{1}{2} \operatorname{SINR}}\right) \text {. }
$$

Where, $\operatorname{erfc}($.$) is the complimentary error function. Fig. 11$ shows the BER achieved with this transmitter for various values of path loss with and without ISI (for comparison). For a path loss of $35 \mathrm{~dB}$ corresponding to a $59 \mathrm{~mm}$ inter-chip link [3], the BER is dominated by the ISI and is around $10^{-10}$.

\section{CONCLUSIONS}

In this paper we present the circuit-level design of a $60 \mathrm{GHz} \mathrm{mm}-$ wave OOK transmitter for inter and intra-chip communications. A bit energy-efficiency of $0.24 \mathrm{pJ} / \mathrm{bit}$ at $16 \mathrm{Gbps}$ is obtained at a low BER of $10^{-10}$ for typical transmission distances. This energy-efficiency

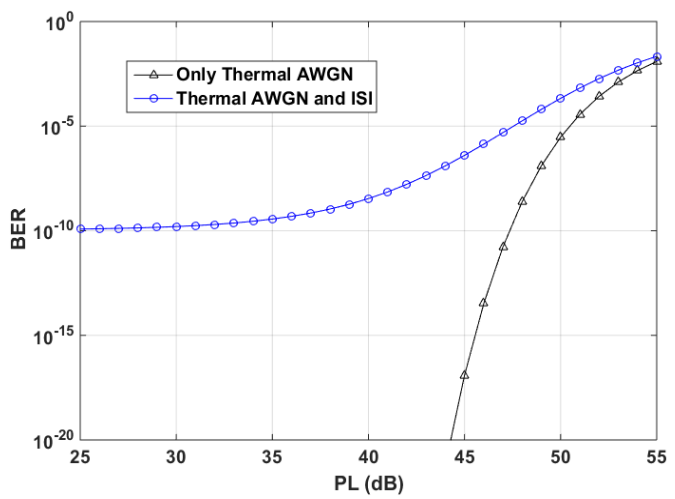

Figure 11: Variation of BER with Path Loss.

makes the mm-wave wireless interconnect competitive with other emerging technologies such as photonic interconnects.

\section{ACKNOWLEDGMENTS}

This work was supported in part by the US National Science Foundation (NSF) CAREER grant CNS-1553264. The authors would also like to thank Avijit Chakraborty, Intel Corp, for his valuable suggestions.

\section{REFERENCES}

[1] J.E. Jaussi, M. Leddige, B. Horine, F. O'Mahony and B. Casper. 2010. Multi-Gbit I/O and interconnect co-design for power efficient links. Electrical Performance of Electronic Packaging and Systems (EPEPS) 2010 IEEE 19th Conference, (October 2010), vol., no., pp.1,4

[2] X. Wu et al. 2104. A Unified Inter/Intrachip Optical Network for Chip Multiprocessors. IEEE TVLSI, (May 2014), vol.22, no.5, pp.1082-1095.

[3] M. S. Shamim, N. Mansoor, R. S. Narde, V. Kothandapani, A. Ganguly and J. Venkataraman. 2017. A Wireless Interconnection Framework for Seamless Inter and Intra-Chip Communication in Multichip Systems. IEEE Transactions on Computers, (March 2017), 66, 3, pp. 389-402.

[4] S. Deb, A. Ganguly, P. P. Pande, B. Belzer, and D. Heo. 2012. Wireless NoC as Interconnection Backbone for Multicore Chips: Promises and Challenges. IEEE JESTCS, (June 2012) vol. 2, no. 2, pp. 228-239.

[5] J. Lee, Y. Huang, Y. Chen, H. Lu, and C. Chang. 2009. A low-power fully integrated $60 \mathrm{GHz}$ transceiver system with $\mathrm{OOK}$ modulation and on-board antenna assembly. IEEE Int. Solid-State Circuits Conf. Tech. Dig, (February 2009) pp. 316-317.

[6] W. Chen et al. 2009. A 6-Gb/s wireless inter-chip data link using 43-GHz transceiver and bond-wire antennas. IEEE J. Solid-State Circuits, (October 2009). vol. 44 , no. 10 , pp. $2711-2721$.

[7] K. Kawasaki et al. 2010. A millimeter-wave intra-connect solution. IEEE J. SolidState Circuits, (December 2010). vol. 45, no. 12, pp. 2655-2666.

[8] E. Juntunen et al. 2010. A 60-GHz 38-pJ/b 3.5-Gb/s $90 \mathrm{~nm}$ CMOS OOK digital radio. IEEE Trans. Microw. Theory Techn., (February 2010). vol. 58, no. 2, pp. 348-355.

[9] Y. Tanaka et al. 2012. A versatile multi-modality serial link. IEEE Int. Solid-State Circuits Conf. Tech. Dig., (February 2012). pp. 332-333.

[10] C. W. Byeon, C. H. Yoon and C. S. Park. 2013. A 67-mW 10.7-Gb/s 60-GHz OOK CMOS transceiver for short-range wireless communications. IEEE Trans. Microwave Theory Techniques, (September 2013). vol. 61, no. 9, pp. 3391-3401.

[11] X. Yu, et al. 2014. A 1.2-pJ/bit 16-Gb/s 60-GHz OOK Transmitter in 65-nm CMOS for Wireless Network-On-Chip. IEEE Transactions on Microwave Theory and Techniques, (2014). vol. 62, no. 10, pp. 2357-2369.

[12] T. Dellsperger. 2002. Design of a $5 \mathrm{GHz}$ VCO in CMOS. Technical report. Swiss Federal Institute of Technology Zurich, Zurich, Switzerland.

[13] A. Mineyama, et al. 2011. A millimeter-wave CMOS low noise amplifier using transformer neutralization techniques. Proc. Asia-Pacific Microwave Conference, (December 2011). pp. 223-226. 